\title{
On residually finite groups satisfying an Engel type identity
}

\author{
Danilo Silveira
}

\begin{abstract}
Let $n, q$ be positive integers. We show that if $G$ is a finitely generated residually finite group satisfying the identity $\left[x, n y^{q}\right] \equiv 1$, then there exists a function $f(n)$ such that $G$ has a nilpotent subgroup of finite index of class at most $f(n)$. We also extend this result to locally graded groups.
\end{abstract}

\section{Introduction}

Let $n$ be a positive integer. We say that a group $G$ is (left) $n$-Engel if it satisfies the identity $\left[y,{ }_{n} x\right] \equiv 1$, where the word $\left[x,_{n} y\right]$ is defined inductively by the rules

$$
[x, 1 y]=x^{-1} y^{-1} x y, \quad\left[x,_{n} y\right]=\left[\left[x,_{n-1} y\right], y\right] \quad \text { for all } n \geq 2 .
$$

A important theorem of Wilson [13, Theorem 2] says that finitely generated residually finite $n$-Engel groups are nilpotent. More specific properties of residually finite $n$-Engel groups can be found for example in a theorem of Burns and Medvedev (quoted below as Theorem 3.5) stating that there exist functions $c(n)$ and $e(n)$ such that any residually finite $n$-Engel group $G$ has a nilpotent normal subgroup $N$ of class at most $c(n)$ such that the quotient group $G / N$ has exponent dividing $e(n)$. The interested reader is referred to the survey [12] and references therein for further results on finite and residually finite Engel groups. The purpose of the present article is to provide the proof for the following theorem.

Theorem 1.1. Let $G$ be a finitely generated residually finite group satisfying the identity $\left[x,{ }_{n} y^{q}\right] \equiv 1$. Then there exists a function $f(n)$

2010 Mathematics Subject Classification. 20F45, 20E26, $20 \mathrm{~F} 40$.

Key words and phrases. Engel element, Engel groups, Residually finite groups, Locally graded groups, Lie Algebras.

Dedicated to Pavel Shumyatsky on the occasion of his 60th birthday. 
such that $G$ has a nilpotent subgroup of finite index of class at most $f(n)$.

A group is called locally graded if every non-trivial finitely generated subgroup has a proper subgroup of finite index. The class of locally graded groups contains locally (soluble-by-finite) groups as well as residually finite groups. We can extend the Theorem 1.1 to the class of locally graded groups.

Corollary 1.2. Let $G$ be a finitely generated locally graded group satisfying the identity $\left[x,_{n} y^{q}\right] \equiv 1$. Then there exists a function $f(n)$ such that $G$ has a nilpotent subgroup of finite index of class at most $f(n)$.

In the next section we describe the Lie-theoretic machinery that will be used in the proof of Theorem 1.1. The proof of the theorem and of the corollary is given in Section 3 .

\section{About Lie algebras}

Let $L$ be a Lie algebra over a field $K$ and $X$ a subset of $L$. By a commutator in elements of $X$ we mean any element of $L$ that can be obtained as a Lie product of elements of $X$ with some system of brackets. If $x_{1}, \ldots, x_{k}, x, y$ are elements of $L$, we define inductively

$$
\left[x_{1}\right]=x_{1} ;\left[x_{1}, \ldots, x_{k}\right]=\left[\left[x_{1}, \ldots, x_{k-1}\right], x_{k}\right]
$$

and $\left[x,{ }_{0} y\right]=x ;\left[x,_{m} y\right]=[[x, m-1=1, y]$, for all positive integers $k, m$. As usual, we say that an element $a \in L$ is ad-nilpotent if there exists a positive integer $n$ such that $\left[x,_{n} a\right]=0$ for all $x \in L$. Denote by $F$ the free Lie algebra over $K$ on countably many free generators $x_{1}, x_{2}, \ldots$. Let $f=f\left(x_{1}, x_{2}, \ldots, x_{n}\right)$ be a non-zero element of $F$. The algebra $L$ is said to satisfy the identity $f \equiv 0$ if $f\left(l_{1}, l_{2}, \ldots, l_{n}\right)=0$ for any $l_{1}, l_{2}, \ldots, l_{n} \in L$.

The next theorem represents the most general form of the Lietheoretical part of the solution of the Restricted Burnside Problem [15, 17, 18. It was announced by Zelmanov in [15. A detailed proof can be found in $\mathbf{1 8}$.

Theorem 2.1. Let $L$ be a Lie algebra over a field and suppose that $L$ satisfies a polynomial identity. If $L$ can be generated by a finite set $X$ such that every commutator in elements of $X$ is ad-nilpotent, then $L$ is nilpotent. 
2.1. Associating a Lie ring to a group. Let $G$ be a group. A series of subgroups

$$
G=G_{1} \geq G_{2} \geq \ldots
$$

is called an $N$-series if it satisfies $\left[G_{i}, G_{j}\right] \leq G_{i+j}$ for all $i, j \geq 1$. Obviously any $N$-series is central, i.e. $G_{i} / G_{i+1} \leq Z\left(G / G_{i+1}\right)$ for any $i$. Let $p$ be a prime. An $N$-series is called $N_{p}$-series if $G_{i}^{p} \leq G_{p i}$ for all $i$. Given an $N$-series $(*)$, let $L^{*}(G)$ be the direct sum of the abelian groups $L_{i}^{*}=G_{i} / G_{i+1}$, written additively. Commutation in $G$ induces a binary operation $[\cdot, \cdot]$ in $L^{*}(G)$. For homogeneous elements $x G_{i+1} \in L_{i}^{*}, y G_{j+1} \in L_{j}^{*}$ the operation is defined by

$$
\left[x G_{i+1}, y G_{j+1}\right]=[x, y] G_{i+j+1} \in L_{i+j}^{*}
$$

and extended to arbitrary elements of $L^{*}(G)$ by linearity. It is easy to check that the operation is well-defined and that $L^{*}(G)$ with the operations + and $[\cdot, \cdot]$ is a Lie ring. If all quotients $G_{i} / G_{i+1}$ of an $N$-series $(*)$ have prime exponent $p$ then $L^{*}(G)$ can be viewed as a Lie algebra over the field with $p$ elements. In the important case where the series $(*)$ is the $p$-dimension central series (also known under the name of Zassenhaus-Jennings-Lazard series) of $G$ we write $D_{i}=D_{i}(G)=\prod_{j p^{k} \geq i} \gamma_{j}(G)^{p^{k}}$ for the $i$-th term of the series of $G$, $L(G)$ for the corresponding associated Lie algebra over the field with $p$ elements and $L_{p}(G)$ for the subalgebra generated by the first homogeneous component $D_{1} / D_{2}$ in $L(G)$. Observe that the $p$-dimension central series is an $N_{p}$-series (see [5, p. 250] for details).

The nilpotency of $L_{p}(G)$ has strong influence in the structure of a finitely generated pro- $p$ group $G$. The proof of the following theorem can be found in [4, 1.(k) and 1.(o) in Interlude A].

Theorem 2.2. Let $G$ be a finitely generated pro-p group. If $L_{p}(G)$ is nilpotent, then $G$ is p-adic analytic.

Let $x \in G$ and let $i=i(x)$ be the largest positive integer such that $x \in D_{i}$ (here, $D_{i}$ is a term of the $p$-dimensional central series to $G$ ). We denote by $\tilde{x}$ the element $x D_{i+1} \in L(G)$. We now quote two results providing sufficient conditions for $\tilde{x}$ to be ad-nilpotent. The following lemma was established in [6, p. 131].

Lemma 2.3. For any $x \in G$ we have $(\operatorname{ad} \tilde{x})^{p}=\operatorname{ad}\left(\widetilde{x^{p}}\right)$.

Corollary 2.4. Let $x$ be an element of a group $G$ for which there exists a positive integer $m$ such that $x^{m}$ is n-Engel. Then $\tilde{x}$ is adnilpotent.

The following theorem is a particular case of a result that was established by Wilson and Zelmanov in [14]. 
Theorem 2.5. Let $G$ be a group satisfying an identity. Then for each prime number $p$ the Lie algebra $L_{p}(G)$ satisfies a polynomial identity.

\section{Proof of the main theorem}

The following useful result is a consequence of [13, Lemma 2.1] (see also [11, Lemma 3.5] for details).

Lemma 3.1. Let $G$ be a finitely generated residually finite-nilpotent group. For each prime $p$ let $J_{p}$ be the intersection of all normal subgroups of $G$ of finite p-power index. If $G / J_{p}$ has a nilpotent subgroup of finite index of class at most $c$ for each $p$, then $G$ also has a nilpotent subgroup of finite index of class at most $c$.

Proof. It follows from proof of [11, Lemma 3.5] that there exists a finite set of primes $\pi$ such that $G$ embeds in the direct product $\prod_{p \in \pi} G / J_{p}$. We will identify $G$ with its images in direct product. By hypothesis, for any $p \in \pi, G / J_{p}$ contains a nilpotent subgroup of finite index $H_{p}$ with class at most $c$. Set $H=\cap_{p \in \pi} H_{p}$. Thus, $G \cap H$ has finite index in $G$ and has nilpotency class at mos $c$, which completes the proof.

Recall that a group is locally graded if every non-trivial finitely generated subgroup has a proper subgroup of finite index. Note that the quotient of a locally graded group need not be locally graded, since free groups are locally graded (see [10, 6.1.9]), but no finitely generated infinite simple group is locally graded. However, the following results give a sufficient conditions for a quotient to be locally graded (see [7] for details).

Lemma 3.2. Let $G$ be a locally graded group and $N$ a normal locally nilpotent subgroup of $G$. Then $G / N$ is locally graded.

Let $p$ be a prime and $q$ be a positive integer. A finite $p$-group $G$ is said to be powerful if and only if $[G, G] \leq G^{p}$ for $p \neq 2$ (or $[G, G] \leq G^{4}$ for $p=2$ ), where $G^{q}$ denotes the subgroup of $G$ generated by all $q$ th powers. While considering a pro- $p$ group $G$ we shall be interested only in closed subgroups. So by the commutator subgroup $G^{\prime}=[G, G]$ we mean the closed commutator subgroup, $G^{q}$ means the closed subgroup generated by the $q$ th powers. Similarly to powerful finite $p$-groups, we may define the powerful pro- $p$ groups. For more details we refer the reader to [7, Chapters 2 and 3 ]. In [1] the following useful result for powerful finite $n$-Engel $p$-group was established. 
Lemma 3.3. There exists a function $s(n)$ such that any powerful finite $n$-Engel p-group is nilpotent of class at most $s(n)$.

The proof of Theorem 1.1 will requires the following lemma.

Lemma 3.4. Let $s(n)$ be as in Lemma 3.3. If $G$ is a finitely generated powerful pro-p group satisfying the identity $\left[x,_{n} y^{q}\right] \equiv 1$, then $G^{q}$ has nilpotency class at most $s(n)$.

Proof. Since $G$ satisfies the identity $\left[x,_{n} y^{q}\right] \equiv 1$, we can deduce from [4, Corollary 3.5] that $H=G^{q}=\left\{x^{q} \mid x \in G\right\}$ is a powerful $n$-Engel pro- $p$ group. According to [4, Corollary 3.3], $H$ is the inverse limit of an inverse system of powerful finite $p$-groups $H_{\lambda}$. Lemma 3.3 implies that any group $H_{\lambda}$ has class at most $s(n)$, and so, $H$ has class at most $s(n)$ as well. Finally, by a result due to Zelmanov [16, Theorem 1] saying that any torsion profinite group is locally finite we get that the quotient group $G / H$ is finite. This completes the proof.

The proof of Theorem 1.1 will also require the following result, due to Burns and Medvedev [3].

Theorem 3.5. There exist functions $c(n)$ and $e(n)$ such that any residually finite $n$-Engel group $G$ has a nilpotent normal subgroup $N$ of class at most $c(n)$ such that $G / N$ has exponent dividing $e(n)$.

We are now ready to embark on the proof of our main result.

Proof of Theorem 1.1. For any positive integer $n$ let $s(n)$ and $c(n)$ be as in Lemma 3.3 and Theorem 3.5, respectively. Set $f(n)=$ $\max \{s(n), c(n)\}$. Since $G$ satisfies the identity $\left[x,_{n} y^{q}\right] \equiv 1$ we can deduce from [2, Theorem A] that $H=G^{q}$ is locally nilpotent. According to Lemma 3.2, $G / H$ is locally graded. By Zelmanov's solution of the Restricted Burnside Problem [15, 17, 18], locally graded groups of finite exponent are locally finite (see for example [8, Theorem 1]), and so $G / H$ is finite. Thus $H$ is finitely generated and so it is nilpotent.

By Lemma 3.1, we can assume that $H$ is residually (finite $p$-group) for some prime $p$. If $p$ does not divides $q$, then $H$ is finitely generated residually finite $n$-Engel group. By Theorem 3.5, $H$ contains a nilpotent normal subgroup $N$ of class at most $f(n)$ such that the quotient group $G / N$ has exponent dividing $e(n)$. Thus, we can see that $G / N$ is finite. Thereby, in what follows we can assume that $H$ is residually (finite $p$-group), where $p$ divides $q$.

Set $H=\left\langle h_{1}, \ldots, h_{t}\right\rangle$. Let $L=L_{p}(H)$ be the Lie algebra associated with the $p$-dimensional central series of $H$. Then $L$ is generated by $\tilde{h}_{i}=h_{i} D_{2}, i=1,2, \ldots, t$. Let $\tilde{h}$ be any Lie-commutator in $\tilde{h}_{i}$ and $h$ be 
the group-commutator in $h_{i}$ having the same system of brackets as $\tilde{h}$. Since for any group commutator $h$ in $h_{1} \ldots, h_{t}$ we have that $h^{q}$ is $n$ Engel, Corollary 2.4 shows that any Lie commutator in $\tilde{h}_{1} \ldots, \tilde{h}_{t}$ is adnilpotent. Since $H$ satisfies the identity $\left[x,_{n} y^{q}\right] \equiv 1$, by Theorem 2.5 , $L$ satisfies some non-trivial polynomial identity. According to Theorem $2.1 \mathrm{~L}$ is nilpotent.

Let $\hat{H}$ be the pro- $p$ completion of $H$, that is, the inverse limit of all quotients of $H$ which are finite $p$-groups. Notice that $\hat{H}$ is finitely generated, being $H$ finitely generated. Since the finite $p$-quotients of $H$ are the same as the finite $p$-quotients of $\hat{H}$ by (a) and (d) of $[\mathbf{9}$, Proposition 3.2.2], we get that $L_{p}(\hat{H})=L$. Hence, $L_{p}(\hat{H})$ is nilpotent and so, $\hat{H}$ is a $p$-adic analytic group by Theorem 2.2 . By [4, 1.(a) and 1.(o) in Interlude A], $\hat{H}$ is virtually powerful, that is, $H$ has a powerful subgroup $K$ of finite index. By Lemma 3.4, $K^{q}$ has class at most $f(n)$. Furthermore, it follows from [16, Theorem 1] that group $K / K^{q}$ is finite. Finally, since $H$ is residually- $p$, it embeds in $\hat{H}$. Thus, $H \cap K^{q}$ is a nilpotent subgroup of finite index in $G$ of class at most $f(n)$. This completes the proof.

Proof of Corollary 1.2. Let $f(n)$ be as in Theorem 1.1. It follows from [2, Theorem $\mathrm{C}]$ that $H=G^{q}$ is locally nilpotent. By Lemma 3.2. $G / H$ is a locally graded group. By Zelmanov's solution of the Restricted Burnside Problem, locally graded groups of finite exponent are locally finite. Thus, $G / H$ is finite and so, $H$ is a finitely generated nilpotent group. Since polycyclic groups are residually finite [10. 5.4.17], we can deduce from Theorem 1.1 that $H$ contains a subgroup of finite index and of class at most $f(n)$. The proof is complete.

\section{References}

[1] Abdollahi, A., Traustason, G.: On locally finite $p$-group satisfying an Engel condition. Proc. Amer. Math. Soc. 130, 2827-2836 (2002)

[2] Bastos, R., Shumyatsky, P., Tortora, A., Tota, M.: On groups admitting a word whose values are Engel. Int. J. Algebra Comput. 23 no. 1, 81-89 (2013)

[3] Burns, R.G., Medvedev, Y.:, A note on Engel groups and local nilpotence. J. Austral. Math. Soc. Ser. A 64, 92-100 (1998)

[4] Dixon, J.D., du Sautoy, M.P.F., Mann, A., Segal, D.: Analytic Pro-p Groups. Cambridge University Press, Cambridge (1991)

[5] Huppert, B., Blackburn, N.: Finite Groups II. Springer-Verlag, Berlin (1982)

[6] Lazard, M.: Sur les groupes nilpotents et les anneaux de Lie. Ann. Sci. École Norm. Sup. 71, 101-190 (1954)

[7] Longobardi, P., Maj, M., Smith, H.: A note on locally graded groups. Rend. Sem. Mat. Univ. Padova, 94, 275-277 (1995) 
[8] Macedońska, O.: On difficult problems and locally graded groups. J. Math. Sci. (N.Y.) 142, 1949-1953 (2007)

[9] Ribes L., Zalesskii, P.: Profinite Groups. Springer-Verlag, Berlin, (2010)

[10] Robinson, D.J.S.: A Course in the Theory of Groups. Springer-Verlag, New York (1996)

[11] Shumyatsky, P.: Applications of Lie ring methods to group theory. (2017). Preprint arXiv:1706.07963 [math.RA].

[12] Traustason, G.: Engel groups. Groups St Andrews 2009 in Bath. Volume 2, 520-550, London Math. Soc. Lecture Note Ser. 388, Cambridge Univ. Press, Cambridge (2011)

[13] Wilson, J.S.: Two-generator conditions for residually finite groups. Bull. London Math. Soc. 23, 239-248 (1991)

[14] Wilson, J.S., Zelmanov, E.I.: Identities for Lie algebras of pro- $p$ groups. J. Pure Appl. Algebra 81, 103-109 (1992)

[15] Zelmanov, E.I.: Nil Rings and Periodic Groups. The Korean Math. Soc. Lecture Notes in Math. Seoul (1992)

[16] Zelmanov, E.I.: On periodic compact groups. Israel J. Math. 77, 83-95 (1992)

[17] Zelmanov, E.I.: Lie methods in the theory of nilpotent groups. in Groups '93 Galaway/ St Andrews, Cambridge University Press, Cambridge 567-585 (1995)

[18] Zelmanov, E.I.: Lie algebras and torsion groups with identity. J. Comb. Algebra 1, 289-340 (2017)

Department of Mathematics, Federal University of Goiás, 75704020 Catalão GO, Brazil

E-mail address: sancaodanilo@gmail.com 